\title{
Progresión clínica de la tiroiditis autoinmune en niños y adolescentes atendidos en el Instituto Nacional de Salud del Niño
}

\author{
Clinical progression of autoimmune thyroiditis in children and \\ adolescents treated at the Instituto Nacional de Salud del Niño
}

\author{
Oswaldo Núñez Almache ${ }^{1,2}$, Carlos Del Aguila Villar ${ }^{1,2}$, Eliana Chávez Tejada², \\ Oscar Espinoza Robles², Paola Pinto Ibárcena², Martha Calagua Quispe ${ }^{2}$, \\ Miguel De los Santos La Torre ${ }^{2}$ \\ Facultad de Medicina, Universidad Nacional Federico Villarreal, Lima, Perú. \\ ${ }^{2}$ Servicio de Endocrinologia, Instituto Nacional de Salud del Niño, Lima, Perú.
}

\begin{abstract}
Resumen
Introducción. La tiroiditis autoinmune es la causa más frecuente de hipotiroidismo adquirido en la edad pediátrica. El cuadro clínico al diagnóstico depende de la condición bioquímica en la que se encuentre y el seguimiento es fundamental para adoptar las medidas terapéuticas que garanticen un adecuado crecimiento y desarrollo. Objetivo. Describir la progresión clínica de la tiroiditis autoinmune en niños y adolescentes. Diseño. Estudio de serie de casos, longitudinal y retrospectivo. Lugar. Servicio de Endocrinología, Instituto Nacional de Salud del Niño, Lima, Perú (institución docente). Participantes. Niños y adolescentes con tiroiditis autoinmune. Intervenciones. Sesenta y cinco pacientes con tiroiditis autoinmune (58 mujeres y 7 varones), menores de 18 años, atendidos entre los años 2000 y 2012, tuvieron seguimiento por más de un año, con evaluaciones clínicas y bioquímicas periódicas. Se excluyó del estudio a los portadores de síndromes genéticos o diabetes mellitus tipo 1. Principales medidas de resultados. Progresión clínica de la tiroiditis autoinmune. Resultados. El bocio $(78,5 \%)$ fue el signo predominante y a la vez factor de riesgo para evolucionar a hipotiroidismo establecido (adquirido) en quienes debutaron con hipotiroidismo subclínico. Al ingreso, el $48 \%$ de prepúberes presentó disfunción tiroidea frente al $26,6 \%$ de los púberes. De la población total, $46 \%$ tuvo hipotiroidismo establecido, $26 \%$ hipotiroidismo subclínico y $23 \%$ estado eutiroideo. El $27 \%$ de eutiroideos y $47 \%$ de hipotiroideos subclínicos evolucionaron a hipotiroidismo establecido. No hubo diferencia estadísticamente significativa de los puntajes Z promedio de talla o IMC al diagnóstico o durante el seguimiento. Conclusiones. Los niños y adolescentes con tiroiditis autoinmune que debutan en estado eutiroideo o hipotiroideo subclínico tienen riesgo alto de evolucionar a hipotiroidismo establecido, siendo importante monitorizar periódicamente la función tiroidea para iniciar oportunamente la terapia de reemplazo hormonal.
\end{abstract}

Palabras clave. Tiroiditis, Autoimmune; Tiroiditis, linfocitica; Enfermedad de Hashimoto.

\section{Abstract}

Introduction. Autoimmune thyroiditis is the most common cause of hypothyroidism in children. Clinical diagnosis depends on biochemical condition and monitoring is essential to adopt therapeutic measures and ensure proper growth and development. Objective. To describe the clinical progression of autoimmune thyroiditis in children and adolescents. Design. Retrospective, longitudinal series of cases study. Setting. Department of Endocrinology, Instituto Nacional de Salud del Niño, Lima, Peru (a teaching institution). Participants. Children and adolescents suffering of autoimmune thyroiditis. Interventions. Sixty-five patients -58 women and 7 men- under the age of 18 suffering of autoimmune thyroiditis and admitted between 2000-2012 were followed with clinical and biochemical evaluations over one year; carriers of genetic syndromes or with type 1 diabetes mellitus were excluded. Main outcome measures. Clinical progression of autoimmune thyroiditis. Results. Goiter (78.5\%) was a predominant sign and risk factor for development of acquired hypothyroidism in those who debuted with subclinical hypothyroidism. At admission $48 \%$ of prepubescent infants presented thyroid dysfunction compared with $26.6 \%$ of pubescent subjects. Out of the total population $46 \%$ suffered of acquired hypothyroidism, $26 \%$ subclinical hypothyroidism, and $23 \%$ were euthyroid; $27 \%$ of euthyroid patients and $47 \%$ of subjects with subclinical hypothyroidism evolved into acquired hypothyroidism. There was no statistically significant difference in the mean Z score for height or BMl at diagnosis nor during follow-up. Conclusions. Children and adolescents with autoimmune thyroiditis debuting as euthyroid or with subclinical hypothyroidism had a high risk of developing acquired hypothyroidism. It seems important to monitor thyroid function for timely initiation of hormone replacement therapy.

Keywords. Thyroiditis, Autoimmune; Thyroiditis, lymphocytic; Hashimoto Disease.

An Fac med. 2015;76(4):325-31/ http://dx.doi.org/10.15381/anales.v76i4.11401 


\section{INTRODUCCIÓN}

La tiroiditis autoinmune (TAI), tiroiditis linfocítica crónica o tiroiditis de Hashimoto es la causa más común de hipotiroidismo adquirido en la edad pediátrica y a pesar de su relativa prevalencia, son pocos los reportes sobre su presentación y evolución ${ }^{(1-3)}$. La TAI está estrechamente relacionada con la enfermedad de Graves, ambas con un fondo genético complejo común o predominante en alguna de ellas, habiéndose postulado la participación de genes específicos de la tiroides y entre 20 a 60 genes implicados en el reconocimiento inmunológico, cada uno con pequeño efecto 'disparador' desconocido ${ }^{(4,5)}$. Mientras que en la TAI predomina la respuesta inflamatoria mediada por linfocitos y citoquinas ${ }^{(6,7)}$, en la enfermedad de Graves lo hace la respuesta mediada por anticuerpos; sin embargo, ambas pueden encontrarse superpuestas en algunos pacientes ${ }^{(8)}$.

Se han descrito dos variantes de la TAI: la forma bociógena (descrita por Hashimoto) y no bociógena (también llamada tiroiditis atrófica o mixedema primario). Suele ser más frecuente en el sexo femenino y se halla asociada a historia familiar de enfermedad tiroidea autoinmune (tanto TAI como Graves) en 30 a 40\% de los pacientes. La edad más frecuente de presentación es la adolescencia, pero puede presentarse a cualquier edad, incluso en la infancia ${ }^{(9)}$. La prevalencia de TAI se encuentra incrementada en pacientes con diabetes mellitus tipo 1, 20\% de los cuales tienen anticuerpos antitiroideos positivos y $5 \%$ TSH elevada ${ }^{(10)}$. Además, puede estar presente como parte de los síndromes autoinmunes poliglandulares, en $10 \%$ de los pacientes del tipo 1 , asociada a candidiasis mucocutánea, hipoparatiroidismo e insuficiencia suprarrenal como resultado de una mutación en el gen AIRE (regulador autoinmune) ${ }^{(11)}$, o en el tipo 2 (síndrome de Schmidt), donde suele presentarse en la adolescencia asociado a diabetes mellitus con o sin insuficiencia suprarrenal ${ }^{(12)}$. Su incidencia también suele estar incrementada en cier- tas anomalías cromosómicas, como los síndromes de Down, Turner, Noonan y Klinefelter; y con urticaria crónica, vitíligo y glomerulonefritis por complejos inmunes ${ }^{(13-16)}$.

Los anticuerpos antitiroideos (AAT) antiperoxidasa-ATPO y antitiroglobulina-Atg son detectables en 95\% de los pacientes con TAI, siendo TPO el más sensible como marcador de daño tiroideo autoinmune. También se detectan, en pequeña proporción, anticuerpos contra el receptor del TSH de tipo estimulatorio, observándose clínica de hipertiroidismo (Hashitoxicosis). Los anticuerpos bloqueadores del receptor de TSH están presentes en menos del 10\% de los pacientes, pero se hallan asociados a marcado hipotiroidismo con y sin bocio; y se ha sugerido que su presencia a títulos altos en forma persistente en mujeres adolescentes gestantes puede predecir riesgo alto de hipotiroidismo congénito transitorio en sus hijos ${ }^{(17)}$. El rol de los AAT en el bocio, resultante de la infiltración linfocítica crónica, es controversial ${ }^{(18)}$.

Clínica y bioquímicamente, los niños $o$ adolescentes con TAI pueden encontrarse eutiroideos ${ }^{(19)}$, con hipotiroidismo primario subclínico (definido como una elevación de la concentración de TSH y una concentración de T4 libre normal, en ambos casos teniendo por referencia los rangos establecidos por el laboratorio) ${ }^{(20)}$, o hipotiroidismo establecido; o en algunos casos experimentar una fase inicial tirotóxica debida a la descarga de T4 y T3 preformados o a la estimulación tiroidea por anticuerpos estimulantes del receptor de TSH.

Un hipotiroidismo establecido tendrá repercusión sobre el crecimiento y desarrollo físico e intelectual del niño y adolescente, por lo que tras su identificación debe iniciarse terapia de sustitución con levotiroxina sódica, mientras que en el hipotiroidismo subclínico puede verse afectado el estirón puberal y la terapia de sustitución se considerará en forma individual (21-23).

Los estudios de seguimiento a largo plazo de niños con TAI sugieren que, mientras la mayoría de quienes debutan con hipotiroidismo establecido se mantiene en ese estado, quienes lo hacen en estado compensado pueden tener recuperación espontánea de la función tiroidea o desarrollar hipotiroidismo durante el seguimiento de la evolución de la enfermedad (24-28).

En nuestro medio no hay reportes sobre esta patología en niños y adolescentes. El INSN, por su servicio de Endocrinología, es el principal centro de referencia nacional para el manejo de la patología tiroidea. En esta condición, estimamos necesario dar a conocer la evolución del TAI en nuestra población, contribuyendo al conocimiento de una enfermedad que debe ser detectada a tiempo para evitar el compromiso del crecimiento y desarrollo de niños $\mathrm{y}$ adolescentes.

El objetivo de la presente investigación es describir la progresión clínica de la tiroiditis autoinmune en niños y adolescentes atendidos en el servicio de endocrinología del Instituto Nacional de Salud del Niño durante el período 2000 a 2012.

\section{MÉTODOS}

Se presenta un estudio observacional, longitudinal, de serie de casos seguidos retrospectivamente. Se realizó en pacientes atendidos en el servicio de Endocrinología del INSN entre los años 2000 y 2013. Se revisaron las historias clínicas de todos los pacientes menores de 18 años con diagnóstico de tiroiditis autoinmune, atendidos en el servicio de Endocrinología durante el período señalado.

Se incluyó las historias clínicas de quienes contaban con determinación de anticuerpos antitiroideos -AAT(antitiroglobulina y/o antiperoxidasa), determinación sérica de TSH, T4 total o libre, más evaluación clínica al diagnóstico y seguimiento durante un año o más después del diagnóstico. Se excluyó los pacientes portadores de síndromes genéticos (Down y Turner) o de diabetes mellitus tipo 1 . 
El diagnóstico de TAI fue establecido por evolución clínica reconocida de tiroiditis autoinmune (fase corta de hipertiroidismo con posterior desarrollo de hipotiroidismo primario adquirido, o hipotiroidismo primario adquirido en niños o adolescentes con historia familiar de tiroiditis autoinmune) asociada o no a incremento del volumen de la glándula tiroidea (bocio) y función tiroidea anormal; o por la presencia de AAT positivos; o finalmente por hallazgo de tiroiditis autoinmune en estudio citológico luego de biopsia aspiración con aguja fina (BAAF).

De la historia clínica se obtuvo datos sobre el motivo de consulta o referencia, antecedentes personales y familiares, antropometría (peso, talla e IMC-peso/talla ${ }^{2}$ ) expresada en desviaciones estándar de acuerdo a las tablas y gráficos de los Centros para Control de Enfermedades de los EEUU de Norteamérica (CDC) ${ }^{(29,30)}$, y el estadio de desarrollo puberal según la clasificación de Tanner ${ }^{(31,32)}$. Se definió bocio como el incremento del volumen del lóbulo lateral de la tiroides mayor al tamaño de la falange distal del primer dedo de la mano del examinado $(>1,0){ }^{(33-36)}$. La determinación de los AAT fue realizada por ELISA y considerada positiva tanto por la elevación de ATPO como de ATg, de acuerdo a los patrones de referencia.

La función tiroidea fue evaluada por determinación de niveles séricos de tirotropina (TSH), con rangos de referencia de 0,4 a 5,0 mUI/L, y tiroxina (T4) total (T4t) o libre (T4-L) con rangos de referencia de 10,5 a $25,7 \mathrm{pmol} / \mathrm{L}$ o 0,8 a $1,8 \mathrm{ng} / \mathrm{dL}$.

De acuerdo a los resultados, los pacientes fueron categorizados como eutiroideos - Eu - (TSH y T4-L normal), portadores de hipotiroidismo subclínico - Hsc - (TSH aumentada y T4-L normal), hipotiroidismo establecido - He - (TSH aumentado y T4-L disminuido), hipertiroidismo subclínico - HTsc - (TSH disminuida con T4-L normal) e hipertiroidismo - HT - (TSH disminuida con T4-L aumentada).
La edad ósea se determinó según el método Tanner Withehouse 2 (TW2) y fue reportado por los médicos de servicio de radiología del INSN. A algunos pacientes se les realizó estudio ecográfico de la tiroides o gammagrafía tiroidea con captación de $\mathrm{I}_{131}$, en la mayoría de casos en centros extrainstitucionales.

El tratamiento en caso de hipotiroidismo subclínico o establecido fue con levotiroxina sódica (LT4) y su monitorización se reporta con los resultados de las determinaciones de TSH y T4-L cada seis meses, durante el seguimiento, aunque se realizó ajustes en la dosificación para mantener un adecuado reemplazo hormonal, en cualquier momento del seguimiento.

Los datos fueron ingresados en una hoja de cálculo del programa Excel 2007 y luego transferidos para su análisis estadístico al paquete SPSS 15.0. Se determinaron las características epidemiológicas de esta población, además de factores de riesgo asociados a tiroidi- tis autoinmune crónica y se evaluó la significancia estadística de los puntajes $Z$ de talla e IMC, así como la variación de los AAT y el estado de función tiroidea durante el seguimiento.

\section{RESULTADOS}

Un total de 65 pacientes (58 mujeres y 7 varones) con diagnóstico de TAI cumplieron los criterios de inclusión y fueron seguidos por un tiempo promedio de 4,24 años (rango: 1,1 a 9,1).

La distribución porcentual del motivo de consulta de los pacientes, al momento del diagnóstico, fue 55,5\% por bocio (forma Hashimoto), 17,5\% por retardo del crecimiento, $9,5 \%$ por obesidad, $4,8 \%$ por signos de hipertiroidismo, 3,2\% por trastornos menstruales, $8 \%$ por diversos motivos, incluido el antecedente familiar de patología tiroidea, y en un solo caso por cuadro clínico típico de hipotiroidismo.

Tabla 1. Características de la población al momento del diagnóstico de TAl.

\begin{tabular}{lc} 
Género & $\mathrm{n}(\%)$ \\
Femenino & $58(89,2)$ \\
Masculino & $7(11,8)$ \\
Edad al diagnóstico (rango) & $9,81(3 \mathrm{a} 15,5)$ \\
Estadio puberal & \\
Prepúber & $28(43,1)$ \\
Púber & $37(56,9)$ \\
$\quad$ Estadio II & 17 \\
Estadio III & 9 \\
Estadio IV & 6 \\
Estadio V & 5 \\
Historia familiar de patología tiroidea & $22(33,8)$ \\
Historia familiar de otras enfermedades autoinmunes & $13(20)$ \\
Antecedentes patológicos de atopia y/o autoinmunidad & $20(30,8)$ \\
Tiempo promedio de enfermedad (meses) & 10 \\
Bocio & $51(78,5 \%)$ \\
Difuso & $44(86,3 \%)$ \\
Nodular & $07(13,7 \%)$ \\
Estado hormonal & \\
Eutiroideo (Eu) & $15(23,1 \%)$ \\
Hipotiroidismo subclínico (Hsc) & $17(26,2 \%)$ \\
Hipotiroidismo establecido (He) & $30(46,2 \%)$ \\
Hipertiroidismo subclínico (HTsc) & $1(1,5 \%)$ \\
Hipertiroidismo (HT) & $2(3,0 \%)$ \\
\hline
\end{tabular}


Tabla 2. Características de la población estudiada, según estado bioquímico al diagnóstico.

\begin{tabular}{|c|c|c|c|c|c|}
\hline & Eutiroideo & $\begin{array}{l}\text { Hipotiroidismo } \\
\text { subclínico }\end{array}$ & $\begin{array}{l}\text { Hipotiroidismo } \\
\text { establecido }\end{array}$ & $\begin{array}{l}\text { Hipertiroidismo } \\
\text { subclínico }\end{array}$ & Hipertiroidismo \\
\hline Sexo (mujer:varón) & $15: 0$ & $14: 3$ & $26: 4$ & $1: 0$ & $2: 0$ \\
\hline Edad & $\begin{array}{c}11 \\
(6,4 \text { a } 14)\end{array}$ & $\begin{array}{c}10,1 \\
(6,6 \text { a } 17)\end{array}$ & $\begin{array}{c}10,3 \\
(3 \text { a } 15,8)\end{array}$ & 7,4 & $\begin{array}{c}14,7 \\
(12,4 \text { a } 16,9)\end{array}$ \\
\hline Prepúber/Púber & $4 / 11$ & $7 / 12$ & $11 / 17$ & $1 / 0$ & $0 / 2$ \\
\hline Bocio & $100 \%$ & $78,9 \%$ & $39,3 \%$ & $100 \%$ & $100 \%$ \\
\hline Bocio nodular & $6,6 . \%$ & $0 \%$ & $7,1 \%$ & $0 \%$ & $0 \%$ \\
\hline Talla_Z & $\begin{array}{c}0,9 \\
(-1,9 \text { a } 2,0)\end{array}$ & $\begin{array}{c}1,0 \\
(-1,5 \text { a } 3,0)\end{array}$ & $\begin{array}{c}0,1 \\
(-3,7 \text { a } 3,0)\end{array}$ & 0,6 & 0,6 \\
\hline IMC_Z & $\begin{array}{c}0,5 \\
(-1,3 \text { a } 1,7)\end{array}$ & $\begin{array}{c}0,5 \\
(-1,5 \text { a } 2,4)\end{array}$ & $\begin{array}{c}1,0 \\
(-0,5 \text { a } 2,8)\end{array}$ & 1,6 & 0,2 \\
\hline AAT positivos & $86,6 \%$ & $100 \%$ & $100 \%$ & $100 \%$ & $100 \%$ \\
\hline Tratamiento con LT4 & $53 \%$ & $79 \%$ & $100 \%$ & -- & -- \\
\hline LT4 (ug/kg) & 0,6 & 0,9 & 2,1 & 0 & 0 \\
\hline
\end{tabular}

Las características de la población al momento del diagnóstico se presentan en la tabla 1, destacándose que la mayoría fueron púberes $(56,91 \%)$ y que la relación entre mujeres y varones fue 8,3:1 (8,3:1 en pre-púberes y 8,2:1 en púberes). Un tercio de la población reportó historia familiar de patología tiroidea y $30 \%$ antecedente patológico de atopía o autoinmunidad ( 2 con diagnóstico de ARJ). Además, predominaron clínicamente el bocio (volumen tiroideo promedio de $1,75 \mathrm{~N}$, hasta un máximo de $2,5 \mathrm{~N}$ ) y hormonalmente el estado de hipotiroidismo primario establecido.

La edad ósea solo fue realizada en $35,4 \%$ (23) de la población de estudio, y en menor número durante el seguimiento; de similar forma, la talla paterna fue obtenida en pocos casos, dificultando la obtención de la talla diana, lo que no ha permitido un mayor análisis de las variables.

La ecografía y gammagrafía tiroideas, al diagnóstico, fueron realizadas en $24,6 \%$ de los casos; por ultrasonografía se demostró bocio difuso en $61,5 \%$ y nodular en $38,5 \%$; mientras que por gammagrafía, $69 \%$ presentó bocio difuso (4 hipercaptadores, 2 hipocaptadores y 4 normocaptadores), $8 \%$ bocio nodular y $23 \%$ fueron normales.
El control periódico de los AAT no fue regular, dificultando su evaluación, de acuerdo al estado bioquímico, durante el seguimiento.

El 21,5\% (14) de los pacientes presentó TAI sin bocio; de ellos, 71,4\% (10) tenía hipotiroidismo establecido y $28,6 \%$ (4) hipotiroidismo subclínico. El $78,5 \%$ tuvo bocio y de acuerdo al volumen el comportamiento hormonal fue como sigue: en $\leq 1,5 \mathrm{~N}$ (19: $7 \mathrm{Eu}, 10$ $\mathrm{Hsc}$ y $2 \mathrm{He}$ ); en $>1,5$ a 2,0 N (18: $6 \mathrm{Eu}$, $3 \mathrm{Hsc}, 7 \mathrm{He}, 1 \mathrm{HTsc}$ y $1 \mathrm{HT}$ ); y en $>2$ (14: $2 \mathrm{Eu}, 11 \mathrm{He}$ y $1 \mathrm{HT})$.

En la tabla 2, se muestra las características clínicas y bioquímicas de la población estudiada de acuerdo al estado bioquímico (hormonal) al momento del diagnóstico.

De las 15 pacientes, todas del sexo femenino, que se encontraban en estado bioquímico eutiroideo (Eu), 13 fueron diagnosticadas por AAT positivos, 1 por BAAF de tiroides y 1 por estudio anatomopatológico luego de hemitiroidectomía por nódulo frío con leve atipia celular por BAAF. De las 13 con AAT positivos, una tuvo diagnóstico ecográfico de bocio nodular, pero la gammagrafía informó bocio difuso normocaptador, mientras otras dos presentaron bocio difuso hipercaptador. Durante la evolución, una paciente pasó a la condición de Hsc, 4 pasaron a He y una desarrolló HTsc a los 6 meses de seguimiento y permaneció en esa condición hasta el corte final del estudio.

De los 17 pacientes (14 mujeres y 3 varones) que debutaron con hipotiroidismo subclínico (Hsc), el 100\% tuvo AAT positivos; $76 \%$ (13) presentó bocio (ninguno nodular) y fue esa la principal razón por la cual el $76 \%$ inició tratamiento con LT4. Durante la evolución, el 17,5\% (3) pasó a condición de $\mathrm{Eu}$, otro $17,5 \%$ permaneció en Hsc y 65\% (11) pasó a He. Respecto al tratamiento, del $24 \%$ que no inició tratamiento con LT4 (4) una pasó a condición de Eu y tres a He; mientras que de las que recibieron LT4, 2 pasaron a la condición de Eu, 3 se mantuvieron en Hsc y 8 pasaron a condición de He. De otro lado, el tratamiento con Lt4 a pacientes con Hsc no modificó el volumen tiroideo durante la evolución.

Los 30 pacientes (26 mujeres y 4 varones) con hipotiroidismo establecido $(\mathrm{He})$, permanecieron en dicha condición y recibieron LT4 según requerimiento.

La paciente que debutó con hipertiroidismo subclínico (HTsc), volvió a la condición de eutiroidea a los 3 años de seguimiento y permaneció así hasta el final del estudio. 
Tabla 3. Evolución del estado bioquímico (hormonal) de la población estudiada, de acuerdo a su condición inicial.

\begin{tabular}{|c|c|c|c|c|c|c|c|c|c|c|}
\hline \multirow[b]{2}{*}{ Diagnóstico } & \multicolumn{5}{|c|}{ Sexto mes } & \multicolumn{5}{|c|}{ Final del estudio } \\
\hline & $\begin{array}{l}\text { Eu } \\
(\%)\end{array}$ & $\begin{array}{l}\text { Hsc } \\
(\%)\end{array}$ & $\begin{array}{l}\mathrm{He} \\
(\%)\end{array}$ & $\begin{array}{l}\text { HTsc } \\
(\%)\end{array}$ & $\begin{array}{l}\text { HT } \\
(\%)\end{array}$ & $\begin{array}{l}\text { Eu } \\
(\%)\end{array}$ & $\begin{array}{l}\text { Hsc } \\
(\%)\end{array}$ & $\begin{array}{l}\mathrm{He} \\
(\%)\end{array}$ & $\begin{array}{l}\text { HTsc } \\
(\%)\end{array}$ & $\begin{array}{l}\text { HT } \\
(\%)\end{array}$ \\
\hline Eutiroideo (15) & $\begin{array}{c}9 \\
(60)\end{array}$ & $\begin{array}{c}1 \\
(6,7)\end{array}$ & $\begin{array}{c}4 \\
(26,6)\end{array}$ & $\begin{array}{c}1 \\
(6,7)\end{array}$ & 0 & $\begin{array}{c}9 \\
(60)\end{array}$ & $\begin{array}{c}1 \\
(6,7)\end{array}$ & $\begin{array}{c}4 \\
(26,6)\end{array}$ & $\begin{array}{c}1 \\
(6,7)\end{array}$ & 0 \\
\hline $\begin{array}{l}\text { Hipotiroidismo } \\
\text { subclínico }(17)^{*}\end{array}$ & $\begin{array}{c}9 \\
(529)\end{array}$ & $\begin{array}{c}4 \\
(23,5)\end{array}$ & $\begin{array}{c}2 \\
(11,8)\end{array}$ & $\begin{array}{c}1 \\
(5,9)\end{array}$ & $\begin{array}{c}1 \\
(5,9)\end{array}$ & $\begin{array}{c}5 \\
(29)\end{array}$ & $\begin{array}{c}4 \\
\text { (24) }\end{array}$ & $\begin{array}{c}8 \\
(47)\end{array}$ & 0 & 0 \\
\hline $\begin{array}{l}\text { Hipotiroidismo } \\
\text { establecido (30)* }\end{array}$ & 0 & 0 & $\begin{array}{c}30 \\
(100)\end{array}$ & 0 & 0 & 0 & 0 & $\begin{array}{c}30 \\
(100)\end{array}$ & 0 & 0 \\
\hline $\begin{array}{l}\text { Hipertiroidismo } \\
\text { subclínico }(1)^{*}\end{array}$ & 0 & 0 & 0 & $\begin{array}{c}1 \\
(100)\end{array}$ & 0 & $\begin{array}{c}1 \\
(100)\end{array}$ & 0 & 0 & 0 & 0 \\
\hline Hipertiroidismo $(2)^{*}$ & 0 & 0 & 0 & 0 & 2 & $\begin{array}{c}1 \\
(50)\end{array}$ & 0 & $\begin{array}{c}1 \\
(50)\end{array}$ & 0 & 0 \\
\hline
\end{tabular}

Eu= eutiroideo, $\mathrm{Hsc}=$ hipotiroidismo subclínico, $\mathrm{He}=$ hipotiroidismo establecido. $\mathrm{HTsc}=$ hipertiroidismo subclínico, HT= hipertiroidismo.

* Algunos recibieron LT4 o tiamazol durante su evolución.

De las dos pacientes con diagnóstico inicial de hipertiroidismo (HT), una volvió a la condición de $\mathrm{Eu}$ al año de seguimiento y permaneció así hasta el final estudio; mientras que la otra pasó a la condición de $\mathrm{He}$ a los 2 años de seguimiento.

En la tabla 4 se muestra la progresión de los puntajes $Z$ de talla e IMC de los pacientes según su estado puberal, observándose que no existió diferencia estadística significativa en ninguno de los dos casos.

\section{DISCUSIÓN}

Se expone retrospectivamente el curso clínico de 65 niños y adolescentes diagnosticados de TAI durante los últimos 10 años en la institución. No existen reportes similares en nuestro medio y, en los últimos años, refiriéndose a características clínicas, solo hay una publicación en la región (37).

Se ha encontrado, al igual que la mayoría de estudios, predominancia del sexo femenino $(8,3: 1)$ y presentación más frecuente durante la pubertad (56,9\%). Sin embargo, en los púberes, a diferencia de lo señalado por de Vries, quien señala mayor riesgo de desarrollar TAI en varones ${ }^{(41)}$ por el aumento en la concentración de estrógenos, no se ha hallado diferencia alguna $(8,2: 1)$. Cuando se determinó la edad de presentación, teniendo en cuenta el estado bioquímico (hormonal) de disfunción tiroidea (Hsc, He, HT e HTsc) frente a eutiroideos (Eu), se halló que en pacientes con disfunción tiroidea la edad promedio al diagnóstico era menor $(9,4 / 11)$ y el número de pacientes en estado prepúber era mayor respecto a los eutiroideos $(48 \% / 26,6 \%)$, similar a lo reportado en un estudio italiano ${ }^{(40)}$. Esto es más significativo si se toma en cuenta que en el estudio no se incluyó pacientes con síndrome de Down o Turner, condiciones que incrementan el riesgo de disfunción tiroidea.

A diferencia de reportes en la literatura, en los que la mayoría de los pacientes fueron asintomáticos al momento del diagnóstico ${ }^{(38-40)}$, se ha hallado al igual que un estudio en Israel ${ }^{(41)}$ y otro en la región andina ${ }^{(37)}$ como principal motivo de consulta la presencia de bocio, situación que podría ser explicada por haber sido habitualmente zonas geográficas bociógenas. Destaca que casi la quinta parte $(17,5 \%)$ acudiera por retardo del crecimiento, en muchos casos debido a que se encontraban en estado bioquímico de $\mathrm{He}$ y aunque el promedio de puntaje $\mathrm{Z}$ para la talla no reflejara talla corta, el amplio rango (- 3DS a 3DS) da muestra del compromiso estatural, más aún si no se pudo precisar su velocidad de crecimiento al diagnóstico. Cabe mencionar que el retardo de crecimiento es el segundo motivo de consulta en la especialidad y que en su guía de evaluación tiene como parte fundamental la evaluación de la función tiroidea, por lo que los hallazgos avalan su realización en la detección de patología tiroidea, especialmente en edad puberal.

Los antecedentes personales y familiares de autoinmunidad estuvieron presentes en un tercio de la población y si bien fueron datos obtenidos de la anamnesis (sin comprobación analítica de laboratorio o patología), son semejantes a lo señalado por otros autores ${ }^{(19,43)}$. Si se tiene en cuenta que se excluyó a pacientes con síndromes genéticos o diabetes mellitus ${ }^{(10-16)}$, gana importancia la valoración de autoin-

Tabla 4. Significancia estadistica de la evolución de los puntajes Z promedio de talla e IMC, según estado puberal, de pacientes con tiroiditis autoinmune.

\begin{tabular}{cccccccc} 
& \multicolumn{3}{c}{ Puntaje Z de talla } & & \multicolumn{3}{c}{ Puntaje Z de IMC } \\
\cline { 2 - 3 } \cline { 8 - 9 } & Inicio & Final & Valor $P$ & & Inicio & Final & Valor $P$ \\
Prepúber & 0,5 & 1,3 & 0,298 & & 1,1 & 0,8 & 0,583 \\
Púber 2-5 & 0,8 & $-0,3$ & 0,956 & & 0,8 & 0,7 & 0.209 \\
\hline
\end{tabular}


munidad tiroidea en la población estudiada y ameritaría un estudio en ese sentido.

La ecografía y gammagrafía tiroidea, a pesar de haber sido realizada a una cuarta parte de la población, permiten señalar que en la mayoría de casos no influyeron en el diagnóstico y tratamiento; por lo que de acuerdo con otros estudios ${ }^{(41)}$, no se les sugiere en la evaluación de pacientes con AAT positivos y sin otra indicación clínica.

Un hecho relevante en el estudio es que la condición hormonal predominante fue la de He, disímil a lo reportado por otros estudios ${ }^{(44)}$ que señalan como predominante el estado Eu; ello podría explicarse por la demora en ser referidos para evaluación o la histórica condición bociógena de algunos sectores de la población. Cuando se evalúa el estado hormonal de acuerdo a la dimensión del bocio, a mayor volumen tiroideo era mayor la presentación de hipotiroidismo establecido, sin que tuviera asociación con el tiempo previo transcurrido hasta el momento del diagnóstico; mientras que, durante la evolución, la disminución del volumen tiroideo en el grupo con $\mathrm{He}$, estuvo asociado al tratamiento con LT4.

De acuerdo a los resultados, el encontrarse en estado eutiroideo al momento del diagnóstico es un factor favorable para permanecer en esa misma condición, ya que solo $25 \%$ evolucionó a $\mathrm{He}$, contrastando con una experiencia europea que reporta hasta un 50\% ${ }^{(45)}$, mientras que debutar en estado de hipotiroidismo establecido es un factor negativo, ya que el $100 \%$ permaneció en similar estado durante la evolución.

En relación a quienes debutaron con hipotiroidismo subclínico, al igual que lo señalado por una revisión ${ }^{(46)}$ se halló que la presencia de bocio es un factor predictivo para desarrollar $\mathrm{He}$, y que el porcentaje que pasa a esa condición es casi tan alto (47\%) como el señalado por el único estudio de esa revisión que reportó un $50 \%{ }^{(47)}$. Este estudio no evaluó la terapéutica en pacientes con Hsc, pero es recomendable adoptar cri- terios para su manejo, como lo señala la literatura ${ }^{(48)}$.

Uno de los aspectos más importantes del seguimiento es el crecimiento de niños y adolescentes. Se evaluó el comportamiento de la talla e IMC, fundamentalmente desde los aspectos del estado de desarrollo puberal y la condición bioquímica de $\mathrm{Hsc}$ e $\mathrm{He}$, ya que la presentación de la enfermedad en fase de hipertiroidismo clínico o subclínico fue muy escasa $(4,6 \%)$ y no permitió mayor análisis. Así, en cuanto al comportamiento de la talla según la condición de prepúber o púber, no se encontró diferencias significativamente estadísticas; sin embargo, en prepúberes la tendencia fue positiva, desviación estándar 0,5 a 1,2, que puede explicarse por el número de niños que alcanzaron la pubertad durante el seguimiento y aquellos que debutaron con $\mathrm{He}$ y revirtieron la disminución en su velocidad de crecimiento una vez iniciada la terapia con LT4; mientras que la tendencia fue negativa en los pacientes púberes, de DE 0,82 a -0,29, lo cual se explica porque los pacientes fueron alcanzando los estadios finales de la pubertad, y la talla diana promedio para nuestra población muestra esa tendencia. En ambos casos, como lo muestran otras experiencias ${ }^{(39-40)}$, lo rescatable es que el promedio de la población estudiada no fue afectada en su crecimiento y que ello dependió del tiempo transcurrido previo al diagnóstico y de la adherencia al tratamiento y seguimiento de los pacientes con TAI.

En relación al IMC promedio al diagnóstico y seguimiento, según estado bioquímico, estado de desarrollo puberal, o tratamiento con LT4, al igual que Özen y col. ${ }^{(49)}$, no se evidenció variación significativa del puntaje $\mathrm{Z}$ promedio del IMC durante el seguimiento. Este hallazgo es importante a tener en cuenta, por cuanto la población suele tener el concepto que el sobrepeso u obesidad se debe a disfunción tiroidea y en muchos casos evaluaciones de la función tiroidea en esos casos pueden mostrar leve hipertirotropinemia que no corresponde a patología tiroidea.
Se concluye que la TAI en niños y adolescentes es más frecuente en mujeres y en estadio púber, pero el presentarse en condición prepúber aumenta el riesgo de disfunción tiroidea; que el signo clínico y la condición bioquímica predominante fueron bocio e $\mathrm{He}$, respectivamente; que el adecuado tratamiento evitó compromiso de la talla e IMC promedio durante la evolución; que un importante porcentaje de pacientes con Hsc evolucionó a $\mathrm{He}$, por lo que es indispensable monitorizar periódicamente su función tiroidea, incluyendo a quienes debutan en estado Eu.

Se sugiere desarrollar estudios controlados y series más grandes para ver la evolución de los AAT como factor pronóstico y la respuesta al tratamiento con LT4 en pacientes con TAI, en especial en aquellos que debutan en condición de Hsc.

\section{REFERENCIAS BIBLIOGRÁFICAS}

1. Rallison ML, Dobyns BM, Keating FR, Rall JE, Tyler $\mathrm{FH}$. Occurrence and natural history of chronic lymphocytic thyroiditis in childhood. J Pediatr. 1975;86(5):675-82.

2. Bachrach LK, Foley TP Jr. Thyroiditis in children. Pediatrics Rev. 1989;11(6):184-9.

3. Foley TP Jr. Hypothyroidism. Pediatr Rev. 2004;25:94-100.

4. Davies TF. Really significant genes for autoimmune thyroid disease do not exist-so how can we predict disease? Thyroid. 2007;17(11):1027-9. doi:10.1089/thy.2007.1526.

5. Brown RS. Autoimmune thyroiditis in childhood. J Clin Res Pediatr Endocrinol. 2013;5 Suppl 1:45-9. doi: 10.4274/jcrpe.855.

6. Stassi G, Di Liberto D, Todaro M, Zeuner A, RicciVitiani L, Stoppacciaro A, et al. Control of target cell survival in thyroid autoimmunity by T-helper cytokines via regulation of apoptotic proteins. Nat Immunol. 2000;1(6):483-8.

7. Phelps E, Wu P, Bretz J, Baker JR. Thyroid cell apoptosis: a new understanding of thyroid autoimmunity. Endocrinol Metab Clin North Am. 2000;29(2):375-88.

8. Vaidya B, Kendall-Taylor P, Pearce SH. The genetics of autoimmune thyroid disease. $\mathrm{J}$ Clin Endocrinol Metab. 2002;87(12):5385-97.

9. Foley TP, Abbassi V, Copeland KC, Draznin MB Brief report: hypothyroidism caused by chronic autoimmune thyroiditis in very young infants. $\mathrm{N}$ Engl J Med. 1994;330(7):466-8.

10. Gilani BB, MacGillivray MH, Voorhess ML, Mills BJ, Riley WJ, MacLaren NK. Thyroid hormone abnormalities at diagnosis of insulin-dependent diabetes mellitus in children. J Pediatr. 1984;105(2):218-22.

11. Betterle C, Greggio NA, Volpato M. Clinical review 93: autoimmune polyglandular syndrome type $1 . \mathrm{J}$ 
Clin Endocrinol Metab. 1998;83(4):1049-55.

12. Soriano RJ, Carrera GE, Sánchez PV, Rubio SY, Guevara LV. Síndrome poliglandular autoinmune tipo II. Rev Med Hosp Gen Mex. 1999;62(2):128-31.

13. Scott HS, Heino M, Peterson P, Mittaz L, Lalioti MD, Betterle $\mathrm{C}$, et al. Common mutations in autoimmune polyendocrinopathycandidiasis-ectodermal dystrophy patients of different origins. Mol Endocrinol. 1998;12(8):1112-9.

14. Wildin RS, Smyk-Pearson S, Filipovich AH. Clinical and molecular features of the immunodysregulation, polyendocrinopathy, enteropathy, X-linked (IPEX) syndrome. J Med genet. 2002;39(8):537-45

15. Leznoff A, Josse RG, Denburg J, Dolovich J. Association of chronic urticaria and angioedema with thyroid autoimmunity. Arch Dermatol. 1983;119(8):636-40

16. O'Regan S, Fong JS, Kaplan BS, Chadarevian JP, Lapointe N, Drummond KN. Thyroid antigen antibody nephritis. Clin Immunol Immunopathol. 1976;6(3):341-6.

17. Feingold SB, Smith J, Houtz J, et al. Prevalence and functional significance of thyrotropin (TSH) receptor blocking antibodies in children and adolescents with chronic lymphocytic thyroiditis. J Clin Endocrinol Metab. 2009;94(12):4742-8. doi: 10.1210/jc.2009-1243.

18. Brown RS. Autoimmune thyroid disease: unlocking a complex puzzle. Curr Opin Pediatr. 2009;21(4):5238. doi: 10.1097/MOP.0b013e32832cf824.

19. Hollowell JG, Stehling NW, Flanders D, Hannon WH, Gunter EW, Spencer CA, Braverman LE. Serum TSH, T4, and thyroid antibodies in the United States population (1988 to 1994): National Health and Nutrition Examination Survey (NHANES III). J Clin Endocrinol Metab. 2002;87(2):489-99.

20. Cooper DS, Biondi B. Subclinical thyroid disease. Lancet. 2012;379(9821):1142-54. doi: 10.1016/ S0140-6736 (11) 60276-6.

21. Jaruratanasirikul $S$, Leethanaporn $K$, Khuntigij $P$, Sriplung $\mathrm{H}$. The clinical course of Hashimoto's thyroiditis in children and adolescents: 6 years longitudinal follow-up. J Pediatr Endocrinol Metab. 2001;14(2):177-84.

22. Svensson J, Ericsson UB, Nilsson P, Olsson C, Jonsson B, Lindberg B, Ivarsson SA. Levothyroxine treatment reduces thyroid size in children and adolescents with chronic autoimmune thyroiditis. J Clin Endocrinol Metab. 2006;91(5):1729-34.

23. Aksoy DY, Kerimoglu U, Okur H, Canpinar H, Karaagaoglu E, Yetgin S, Kansu E, Gedik O. Effects of prophylactic thyroid hormone replacement in euthyroid Hashimoto's thyroiditis. Endocr J. 2005;52(3):337-43.

24. Rallison ML, Dobyns BM, Keating FR, Rall JE, Tyler $\mathrm{FH}$. Occurrence and natural history of chronic lymphocytic thyroiditis in childhood. J Pediatr. 1975;86(5):675-82.

25. Moore DC. Natural course of 'subclinical' hypothyroidism in childhood and adolescence. Arch Pediatr Adolesc Med. 1996;150(3):293-7.

26. Lazar L, Frumkin RB, Battat E, Lebenthal Y, Phillip $\mathrm{M}$, Meyerovitch J. Natural history of thyroid function tests over 5 years in a large pediatric cohort. J Clin Endocrinol Metab. 2009;94(5):1678-82. doi: 10.1210/jc.2008-2615.
27. Radetti G, Gottardi E, Bona G, Corrias A, Salardi S, Loche. The natural history of euthyroid Hashimoto's thyroiditis in children. J Pediatr. 2006;149(6):827-32.

28. Gruñeiro de Papendieck L, lorcansky S, Rivarola MA, Bergadá C. Variation in clinical, hormonal and serological expression of chronic lymphocytic thyroiditis $(C L T)$ in children and adolescents. Clin Endocrinol (Oxf). 1982 Jan;16(1):19-28.

29. Zmarski RJ, Ogden CL, Grummer-Strawn LM. CDC growth charts: United States advanced data from vital and health statistics. NCHS Advance Data Report No 314. Hyatsville, MD: US Department of Health and Human Services. 2000;8(314):1-27.

30. Rosner B, Prineas R, Loggie J, Daniels SR. Percentiles for body mass index in US children 5 to 17 years of age. J Pediatr. 1998;132(2):211-22.

31. Marshall WA, Tanner JM. Variations in the pattern of pubertal changes in girls. Arch Dis Child. 1969;44(235):291-303.

32. Marshall WA, Tanner JM. Variations in patterns of pubertal changes in boys. Arch Dis Child. 1970;45(239):13-23.

33. Perez C, Scrimshaw S, Muñoz A. Technique of endemic goitre surveys. En: Endemic goitre. Geneva: WHO. 1960;44:369-83.

34. DeMaeyer E, Lowenstein F, Thilly C. The control of endemic goitre. Geneva: WHO. 1979. Disponible en: http://www.who.int/iris/handle/10665/40085.

35. Delange F, Bastini S, Benmiloud M, et al. PAHO/ WHO technical group on endemic goitre, cretinism, and iodine deficiency 5th meeting 1983 Lima, Peru. Definition of endemic goitre and cretinism, classification of goitre size and severity of endemias, and survey techniques. En: Dunn J, ed. Towards the eradication of endemic goitre, cretinism, and iodine deficiency. Washington DC: PAHO scientific publication. 1986;502:373-6.

36. Peterson S, Sanga A, Eklöf H, Bunga B. Classification of thyroid size by palpation and ultrasonography in field surveys. Lancet. 2000;355(9198):10610

37. Hayes J P, Montero W. Tiroiditis de Hashimoto en niños y adolescentes con bocio. Rev bol ped 2006;45(2):95-7.

38. Skarpa V, Kappaousta E, Tertipi A, Anyfandakis K Vakaki M, Dolianiti M, Fotinou A, Papathanasiou A. Epidemiological characteristics of children with autoimmune thyroid disease. Hormones. 2011;10(3):207-14.

39. Fava A, Oliverio R, Giuliano S. Clinical evolution of autoimmune thyroiditis in children and adolescents. Thyroid. 2009;19(4):361-7. doi: 10.1089/ thy.2008.0239.

40. Wasniewska M, Corrias A, Salerno M, Mussa A Capalbo D, Messina MF, Aversa T, Bombaci S, De Luca F, Valenzise M. Thyroid function patterns at Hashimoto's thyroiditis presentation in childhood and adolescence: are mainly conditioned by patients' age? Horm Res Paediatr. 2012;78(4):232-6. doi: 10.1159/000343815.

41. de Vries L, Bulvik S, Phillip M. Chronic autoimmune thyroiditis in children and adolescents: at presentation and during long-term follow-up. Arch Dis Child 2009;94(1):33-7. doi: 10.1136/adc.2007.134841.
42. Marwaha RK, Sen S, Tandon N, Sahoo M, Walia RP, Singh S, Ganguly SK, Jain SK. Familial aggregation of autoimmune thyroiditis in first-degree relatives of patients with juvenile autoimmune thyroid disease. Thyroid. 2003;13(3):297-300

43. Segni M, Wood J, Pucarelli I, Toscano V, Toscano $\mathrm{R}$, Pasquino AM. Clustering of autoimmune thyroid diseases in children and adolescents: a study of 66 families. J Pediatr Endocrinol Metab. 2001;14(Suppl 5):1271-5.

44. De Lucca F, Santucci S, Corica D, Pitrolo E, Romeo M, Aversa T. Hashimoto's thyroiditis in childhood: presentation modes and evolution over time. Ital J Pediatr. 2013;39:8. doi: 10.1186/1824-7288-39-8.

45. Radetti G, Gottardi E, Bona G, Corrias A, Salardi S, Loche S. Study group for thyroid diseases of the Italian society for pediatric endocrinology and diabetes (SIEDP/ISPED): the natural history of euthyroid Hashimoto's thyroiditis in children. J Pediatr. 2006;149(6):827-32.

46. Monzani A, Prodam F, Rapa A, Moia S, Agarla V, Bellone S, Bona G. Endocrine disorders in childhood and adolescence. Natural history of subclinical hypothyroidism in children and adolescents and potential effects of replacement therapy: a review. Eur J Endocrinol. 2012;168(1):R1-R11. doi: 10.1530/EJE-12-0656.

47. Jaruratanasirikul S, Leethanaporn K, Khuntigij P, Sriplung $H$. The clinical course of Hashimoto's thryoiditis in children and adolescents: 6 years longitudinal follow-up. J Pediatr Endocrinol Metab. 2001;14(2):177-84.

48. Pearce S H, Brabant G, Duntas L, H, Monzani F, Peeters R, P, Razvi S, Wemeau JL. ETA Guideline: Management of Subclinical Hypothyroidism. Eur Thyroid J. 2013;2(4):215-28. doi: 10.1159/000356507.

49. Özen S, Berk O, Gokşen D, Darcan S. Clinical course of Hashimoto's thyroiditis and effects of levothyroxine therapy on the clinical course of the disease in children and adolescents. J Clin Res Pediatr Endocrinol. 2011;3(4):192-7. doi: 10.4274/ jcrpe. 425 .

Conflicto de intereses: El autor y los coautores declaran no tener ningún conflicto de intereses en la realización del presente estudio.

Fuente de financiamiento: Personal.

Artículo recibido el 20 de febrero de 2015 y aceptado para publicación el 13 de abril de 2015.

\section{Correspondencia:}

Oswaldo Núñez Almache

Av. Naranjal 1712, Los Olivos, Lima - Perú

Teléfono: 999657780

Correo electrónico: isn_ona@hotmail.com;onunez@ insn.gob.pe 